\title{
LA INTERVENCIÓN TERRITORIAL ESTATAL SOBRE LA POBREZA EN ARGENTINA (2008-2015)
}

\section{THE STATE TERRITORIAL INTERVENTION IN POVERTY IN ARGENTINA (2008-2015)}

\section{Claudia Alejandra Martínez Robles*}

\section{RESUMEN}

El presente artículo aborda el modo en que el Estado Nacional Argentino concibe y modula territorialidades específicas en el marco de la política asistencial durante la última década, específicamente, entre los años 2008 y 2015. A partir de un abordaje cualitativo, que combina el análisis documental y entrevistas en profundidad, se indagó sobre la naturaleza de las intervenciones del Estado Argentino en el territorio que tuvieron lugar en el marco del programa nacional denominado "Plan Ahí". La investigación sostiene que el "Plan Ahí" es una intervención de gobierno (Foucault, 2006) que opera como un modelo ordenador de las prácticas estatales, orientadas a transformar la dirección y el sentido de las intervenciones de abordaje territorial, así como, a revertir los efectos de una ausencia estatal supuesta.

PALABRAS CLAVE: TERRITORIO * ESTADO * POLÍTICA PÚBLICA * POBREZA * GOBIERNO

\section{ABSTRACT}

It article addresses the way in which the Argentine National State conceives and regulates specific territorialites within the framework of the social policies of the last decade, more especificaly, between the years 2008 to 2015. Based on a qualitative approach, which combines documental analisys and in-deep interviews, we enquiried into the nature of the State interventions on the territory, that took place following the national programme called "Plan Ahí". The research claims that "Plan Ahí" is a governmental intervention that operates as an organizer model of the state practices, aimed at transforming the direction and meaning of territorial approach interventions, and to reverse the effects of a supposed State absence.

KEYWORDS: TERRITORY * STATE * PUBLIC POLICY * POVERTY * GOVERNMENT

\footnotetext{
* Instituto de Investigaciones Gino Germani, Universidad de Buenos Aires, Argentina.
} claudiamarob@gmail.com 


\section{INTRODUCCIÓN}

En la región Sur de América Latina se han producido, en las últimas décadas, una serie de transformaciones $y$ de propuestas críticas a las reformas neoliberales implementadas en la última década del siglo xx. Argentina formó parte de esta tendencia con sus particularidades. La apremiante situación económica que atravesaba el país durante la década de ajuste estructural, junto a la activación, organización y participación política de los sectores populares y movimientos sociales que pusieron en escena nuevos actores y repertorios de acción, dieron pie a la crisis política e institucional que eclosionó en el 2001.

A partir de este momento, más precisamente desde el año 2003, se produjo no un mero recambio de autoridades, sino la tarea de reconstruir y recomponer el poder político y la legitimidad del Estado. En este escenario, se abre una instancia central para este análisis, a partir de la cual sobrevienen una serie de transformaciones $y$ redireccionamientos orientados a una revalorización de la intervención estatal en materia de política pública. En este sentido, el presente trabajo ${ }^{1}$ apuntó a analizar la dirección y los sentidos específicos que adoptó la política asistencial de corte territorial en este particular momento sociopolítico.

Estas transformaciones no solo hacen posible una intervención territorial específica sino que, al mismo tiempo, son el objetivo central de la política que se analiza en este documento: el Plan Nacional de Abordaje Integral, plan "Ahí en el lugar" o simplemente "Plan Ahí" (PA) $)^{2}$. La pertinencia del PA como referente

$1 \quad$ El artículo presenta los resultados parciales de una investigación más amplia de la tesis doctoral de la autora, la cual se encuentra en proceso. Su elaboración se realiza en el marco del Programa de Estudios Sobre Control Social del Instituto de Investigaciones Gino Germani de la Universidad de Buenos Aires.

2 Desde su nominación, este plan manifiesta la intención del Estado Nacional de hacerse presente en los territorios e indica que su finalidad primera es producir un "estar en el lugar". En consonancia, la revisión de la normativa en la que se basa da cuenta de que el PA se constituye con empírico para el análisis de estas trasformaciones radica en que este programa se propuso como un "modelo", a partir del cual el Estado Nacional debía producir y ordenar sus intervenciones en el territorio en torno a la gestión de la pobreza. A su vez, en tanto su puesta en marcha requirió nuevos desempeños interinstitucionales que se enuncian y esgrimen, política y técnicamente, en oposición al modo de gestión de "lo social" que se llevó adelante durante las décadas previas, así como, al acompasamiento que, por entonces, manifestó la política social y asistencial a los lineamientos y recomendaciones de organismos internacionales.

Por ello, la intención en este estudio fue recuperar y analizar qué racionalidades políticas ${ }^{3}$ orientaron las prácticas estatales que tuvieron lugar en el marco del PA. La reflexión sobre las racionalidades permite entender cómo se configuran, habilitan y cristalizan ciertas conceptualizaciones y justificaciones morales para ejercer el poder. Es decir, hace posible comprender cómo los funcionarios y agentes ministeriales, en su calidad de autoridades de gobierno, definen qué es gobernar, a quiénes se puede gobernar, cómo hacerlo y mediante qué medios ${ }^{4}$. Estas racionalidades, a

el objetivo manifiesto de ampliar y consolidar la presencia del Estado Nacional en los territorios empobrecidos a efectos del retroceso que sufrieron las economías regionales durante la "década neoliberal". Para su instalación, en los territorios se sirvió de la infraestructura y los recursos disponibles que, desde el 2004, produjo el Ministerio de Desarrollo de la Nación (MDSN). En cuanto al diseño de este plan, se delinearon cuatro polos a partir de los cuales se desarrollarían las acciones, a saber: 1) Infraestructura social, 2) Organización social, 3) Productivo o de servicios, y 4) Prestaciones y Servicios.

3 Siguiendo el trabajo de Giavedoni (2012), se entiende que la racionalidad política refiere a "una forma de pensar y ejercer el poder. No alude simplemente a teorizaciones, ideologías o engaños, sino a formas de pensar un problema, de constituirlo, de hacerlo inteligible a través de determinadas características $y$, por ende, formas de actuar sobre él" (p.101).

4 Cabe destacar que la noción de "gobierno" es más amplia. Sintéticamente, se debe mencionar que desde el enfoque de la gubernamentalidad, el 
su vez, se evidencian en los discursos políticos que configuran y expresan ciertos esquemas de la realidad, así como, en los documentos, informes, evaluaciones de agentes estatales, organizaciones político-sociales, expertos, entre otros (Rose, 2007; Giavedoni, 2012; Grondona, 2014).

El programa observado se caracterizó por haber permitido el emplazamiento —en Centros Integradores Comunitarios (cIC) ${ }^{5}$ fijos o móviles-y la circulación de una multiplicidad de agentes de diversos ministerios nacionales ${ }^{6}$ en lugares donde yace el "núcleo duro de la pobreza". Su permanencia en los diversos territorios puso en marcha, de forma coordinada, una serie de programas nacionales y prestaciones en materia de salud, educación, economía social, infraestructura, vivienda, seguridad, acceso a la justicia, entre otros.

Esta coordinación, a su vez, comprendió distintos niveles de intervención gubernamental, es decir, lo local, lo provincial y lo

gobierno refiere a la conducción de la conducta, a la posibilidad de estructurar los campos de acción de los otros y de dejar actuar a los individuos operando sobre los marcos de sus posibles recorridos (Foucault, 2006). Es decir, bajo esta noción se conceptualizan los intentos racionales, y más o menos calculados, de dirigir la acción de los sujetos.

Con el advenimiento del Kirchnerismo, el MDSN impulsa la creación de infraestructura para ampliar su alcance territorial, entre ello, se encuentra la instalación de los cic. Estos consisten en salones de usos múltiples construidos en los barrios con "población vulnerable". Igualmente, en el marco del Plan Ahí se crean los cic móviles, estos eran containers que se establecen en algunos barrios en los que se transportan recursos, se realizan gestiones administrativas $y$ se otorgan prestaciones específicas a los programas. nacional. El despliegue de este conjunto de acciones apuntaron, en primer lugar, a materializar diversos elementos tendientes a instaurar un "nuevo paradigma" de la política pública - tales como el énfasis en la identificación de lazos sociales y dinámicas propias de los grupos sociales locales, la promoción de la participación colectiva y organizada de los actores sociopolíticos situados en los territorios- $-y$, en segundo lugar, a objetivar la idea del "retorno del Estado Nacional".

La promulgación del PA, en abril de 2008 , se produjo en un año en el que se cierra el período de institucionalización política y recomposición económica abierta en el año 2003, dándose inicio a nueva fase a partir del denominado "conflicto del campo". Este conflicto generó una crisis política de gran envergadura, en un contexto de desaceleración del crecimiento económico, en el que se produce un ligero aumento del desempleo (Piva, 2015). En este escenario, desde el gobierno nacional ${ }^{8}$, se impulsaron algunas de las políticas sociales más significativas de los últimos años, tales como la Asignación Universal por Hijo para la Protección Social (AUHPS) ${ }^{9}$ que alcanzó, para el

$7 \quad$ Este enfrentamiento produjo una serie de impugnaciones de la burguesía agraria, que puso en cuestionamiento tanto el estilo político del gobierno, calificado como autoritario, como la delegación de las facultades en el Ejecutivo y una tendencia a la acción directa de los sectores medios urbanos, de fracciones de la clase dominante y de la oposición parlamentaria (Piva, 2015). A este escenario de polarización entre gobierno y oposición se le agrega la pérdida de peso relativo del oficialismo en las cámaras legislativas como resultado del proceso electoral de junio de 2009 , dando lugar a un "bloque de oposición" (Hintze y Costa, 2011).

8 Presidido, en ese entonces, por Cristina Fernández de Kirchner.

9 Decreto 1602/9. Promulgado en octubre de 2009 Esta política amplió la cobertura del régimen de asignaciones invocando el principio de "universalidad" en la medida en la que incorporó a los trabajadores desocupados, monotributistas sociales y quienes se desempeñaban en la economía informal o trabajaban por un ingreso inferior al salario mínimo vital y móvil (Hintze y Costa, 2011). 
año 2014, a 3,3 millones de niños, niñas y adolescentes (Hintze y Costa, 2011; Maneiro, 2015).

Es decir, la creación del "Plan Ahí" se produce simultáneamente a la emergencia de una política orientada hacia una recentralización de la seguridad social ${ }^{10}$. Esto es relevante en tanto que las transformaciones que introducen la AUHPS pueden concebirse como cierta "desterritorialización" de la política social, ya que se produjo sobre el régimen preexistente de asignaciones familiares, distanciándose de las políticas focalizadas ${ }^{11}$ dirigidas sobre los grupos "vulnerables" (usualmente referidos como "barrios" o "comunidades").

Como señalan algunos autores, a finales de los años 80, cuando la "lucha contra la pobreza" se tornó un objetivo central en Latinoamérica (Álvarez, 2006), el territorio fue interpelado para superarla mediante la focalización como herramienta, $y$ la promoción de la competitividad de los territorios. Sin embargo, las crisis económicas y de legitimidad institucional, que eclosionaron en el 2001, mostraron los límites de ese modo de hacer política y el debate sobre políticas públicas comenzó a recuperar como principio la "universalidad", junto con una perspectiva basada en derechos de los ciudadanos (Hinzte y Costa, 2011).

La profundización de las desigualdades, por efecto de las reformas de carácter neoliberal, no solo produjo un cuestionamiento al modelo clásico de políticas públicas neoliberales sino un giro respecto a la centralidad del territorio (en tanto espacio socialmente construido y significado) como el lugar de producción política de las organizaciones sociales; de legitimación de la política estatal y partidaria;

10 Revirtiendo la tendencia que, desde mediados de los años 70 y más drásticamente desde los años 90 , produjo un descentramiento de la matriz de la seguridad social y una centralización de la asistencia, la cual permeó todo campo de la política social más allá de las políticas sociales propiamente dichas (Ayos, 2017).

11 Modo de "selección" tradicionalmente empleado en el marco de las políticas de corte neoliberal. Por el contrario, la AUPH tendió a consolidar un vínculo entre seguridad social, igualdad, solidaridad y el reconocimiento de derechos y garantías para la reproducción de la vida. y como dimensión explicativa para comprender las dinámicas económicas, políticas y sociales (Catenazzi y Quintar, 2009; Vommaro, 2012; Rofman, 2016). Posteriormente, desde el año 2003 , se retoman con renovado vigor los debates sobre el rol regulador del Estado en los procesos de integración territorial y desarrollo.

En este sentido, en los últimos años se ha impulsado, tanto a nivel nacional como municipal, la incorporación del territorio en el diseño de las políticas públicas (Clemente, 2013; Catenazzi, 2015). Precisamente, la "territorialización de la política” se planteó como un eje central que atravesó las políticas sociales del kirchnerismo por lo cual, el programa en estudio resulta de gran pertinencia para reflexionar sobre las mutaciones de sentido que se adoptaron en el marco de las políticas asistenciales de abordaje territorial.

Desde un abordaje cualitativo, que conjugó el análisis de documentos oficiales y entrevistas en profundidad, se indagó las lógicas con las que el Estado Nacional se aproximó $y$ asentó sobre los territorios. La observación permite afirmar que el PA expresa ciertas concepciones sobre la "territorialidad" que intentaron acuñarse durante la década recientemente pasada. A partir de lo mencionado, en el primer punto se presentan algunas consideraciones teórico-metodológicas; en el segundo punto, se expondrá brevemente el debate sobre la territorialización de las políticas sociales $y$ asistenciales en el que se inscribe esta investigación. Seguidamente, se analizan las reorientaciones en los sentidos de las políticas asistenciales de abordaje territorial en el campo de la política asistencial que se evidencian en el PA $y$ las tensiones que se hacen presentes en las formas de concebir la gestión de los territorios. Finalmente, se esbozarán algunas conclusiones a modo de cierre.

\section{CONSIDERACIONES TEÓRICO-METODOLÓGICAS}

El enfoque de este estudio se inscribe en la serie de trabajos sobre las políticas sociales modernas que, en sintonía con el pensamiento de Michael Foucault, se entienden como artes de gobierno específicas sobre determinadas poblaciones, que ponen en juego complejas 
tecnologías, dispositivos y sistemas de enunciaciones (Álvarez, 2006; Murillo, 2008; Dallorso, 2010). Estas investigaciones sostienen que en la racionalidad política neoliberal la cuestión social es enunciada $y$ problematizada en términos de "pobreza" (y no de la desigualdad producto de la relación capital-trabajo). Es decir, las políticas sociales del neoliberalismo se distinguen por la centralidad que cobra la pobreza para los organismos públicos y privados; $y$ la transformación que, en sintonía, se produce en la manera en la que es intervenida la cuestión social (Servio, 2015).

Este enfoque resulta de gran utilidad a los fines de este estudio, ya que permite indagar las formas en que se conciben $y$ gestionan las prácticas estatales $y$ de qué manera se orientan a regular las condiciones de vida y de reproducción de los grupos sociales en condición de pobreza. En pocas palabras, en el presente artículo, el PA será problematizado como una intervención gubernamental ${ }^{12}$ que produce una territorialidad ${ }^{13}$ específica en el marco de la política asistencial. De aquí que el objetivo de este texto sea analizar qué orientaciones tomaron las intervenciones estatales de abordaje territorial —entre los años 2008 y 2015- producidas en el marco del PA. El análisis se desprende de una investigación empírica más amplia y se realiza en base a un enfoque metodológico cualitativo (Vasilachis de Gialdino, 2015), en el que se conjugan técnicas de investigación documental con entrevistas en profundidad a personal funcionario $y$ técnico vinculados a la formulación de la política analizada y a los

12 Se entenderá por intervenciones gubernamentales, aquellas prácticas de gobierno que se caracterizan por estar direccionadas hacia un problema social específico, $y$ que se sostienen a partir de un saber versado $y$ legitimado acerca de la esfera o la cuestión sobre la que apuntan (Dallorso, 2010; Giavedoni, 2012).

13 Específicamente, a efectos de este trabajo, se retomará la noción de territorialidad de Sack (1986). Este autor sostiene que la territorialidad consiste en "el intento por parte de un individuo o grupo de afectar, influenciar o controlar personas, fenómenos y relaciones a través de la delimitación y afirmación del control sobre un área geográfica. Este área será llamada territorio”(p. 21) . "operadores" que llevaron a cabo las tareas previstas por los ministerios y que tuvieron contacto directo con los territorios de intervención $y$ la población objetivo definida por el PA.

\section{DEBATES SOBRE LA TERRITORIALIZACIÓN DE LAS POLÍTICAS SOCIALES Y ASISTENCIALES}

En Argentina, las intervenciones territoriales en el marco de la política social y asistencial ${ }^{14}$ del Estado no son un emergente de esta última década ${ }^{15}$. Sin ir más lejos, durante la década de los 90, el "territorio" y las políticas territoriales de carácter asistencialista y focalizado ${ }^{16}$ cobraron centralidad (Grassi, 2003; Merklen, 2000; Svampa, 2005). A su vez, con la emergencia de movimientos sociales de corte territorial, tales como, piqueteros y asambleas barriales, que incorporaron como una nueva cuestión la construcción de "poder territorial" desde abajo, o de poder desde los márgenes (Svampa, 2005; Varela, 2010), la "territorialización de la política” se tornó preponderante como dimensión de las intervenciones estatales $y$ de los estudios sociopolíticos.

14 Como se sabe, la definición de política social permite distinguir diversas formas de intervención en función del sujeto al que se orienta. Mientras una modalidad de política social puso tradicionalmente en marcha un complejo de seguridades derivadas de la relación salarial formal; otra modalidad, definida como asistencia, apunta a los sujetos que presentan una escasa o nula inserción en el mercado de trabajo, y que en ese sentido se encuentran desprotegidos. Si bien, estas modalidades de intervención sobre la cuestión social son modalidades permanentes y no modelos históricos sucesivos, ambas se inscriben en historias y tradiciones diferentes tal y como sostienen Claudia Danani y Susana Hintze (2014).

15 Contrariamente, a la percepción de algunas personas funcionarias estatales entrevistadas.

16 Se entiende como política social asistencial a aquellas intervenciones secundarias del ingreso, destinadas a atender a sujetos de nula o escasa integración a la condición salarial. Por otra parte, por focalización se hace referencia a la forma en la instalación de una mayor selectividad en la política pública basada en la acreditación de la situación de pobreza como condición para la percepción de la asistencia (Soldano y Andrenacci, 2006). 
Dentro de los estudios sobre la territorialización de la política, se encuentran distintos puntos de vista $y$ abordajes; sin embargo, pese a su heterogeneidad, se distinguen dos matrices de pensamiento. En primer lugar, los estudios que toman como punto de partida la transformación radical del denominado "mundo del trabajo" (proceso que también ha sido denominado como "desafiliación"). Estos trabajos sostienen que, después de suscitada la última dictadura militar en Argentina, la acción colectiva de los sectores populares se habría desplazado desde la fábrica hacia los barrios. En segundo lugar, las investigaciones que cuestionan esta tesis afirman, por el contrario, que el barrio y la fábrica no son espacios separados y autónomos, sino que ambos constituyen terrenos de ejercicio de la política del personal trabajador. En estos espacios, los procesos de politización y despolitización se desarrollan de una manera discordante, generando configuraciones particulares en sendos lugares (Varela, 2010). En otras ocasiones, igualmente, la noción de territorialización aparece para señalar la importancia que cobró el territorio local, como el lugar en el que se despliegan acciones de protesta social.

Ahora bien, cabe destacar que este análisis no parte de conceptualizaciones prefijadas sobre el "territorio". Antes bien, interesa abordarlo como el producto de una multiplicidad de intervenciones en la cotidianeidad de la población, entre las cuales tienen lugar las del Estado. Al mismo tiempo, no se pierde de vista que las relaciones de poder implícitas o explícitas, materiales o simbólicas que construyen los territorios como espacios diferenciales, se producen a partir de la articulación de percepciones y concepciones particulares de los sujetos, los grupos y las clases sociales que los conforman (Manzanal, 2016).

En consecuencia, se rastreó cómo se identifican y delimitan los ámbitos de incumbencia del Plan, lo que le es "propio" y lo que es "ajeno" a dichos territorios; cuáles son las claves y los objetivos a partir de los que se habilitan las intervenciones sobre tales espacios $y$, vinculado a ello, qué tensiones o disputas se evidencian por el control de estos. Estas premisas han permitido arribar a las principales concepciones en torno al territorio que se pusieron en juego en el PA.

Si bien, la emergencia de este programa específico se produce en el año 2008, la revisión del material documental producido por los distintos ministerios entre los años 2008 y 2015 (Ministerio de Desarrollo Social de la Nación, 2007, 2009, 2010, 2013a, 2013b y 2013c; Jefatura de Gabinete de la Nación, 2004, 2010a y 2010b; Ministerio de Educación de la Nación, 2014; Ministerio del Interior de la Nación, 2004) permite afirmar que las conceptualizaciones sobre el territorio expuestas en el PA expresan una síntesis, esto es, una condensación de concepciones sobre lo territorial que venían esbozándose desde el MDSN a partir del año 2003. Simultáneamente, es posible reconocer en estas prácticas una serie de reflexiones teóricas sobre los territorios propios al acervo de las ciencias sociales.

\section{PERCEPCIONES SOBRE LA INTERVENCIÓN TERRITORIAL DEL ESTADO NACIONAL}

Los documentos rastreados, emitidos por los distintos ministerios, reflejan una concepción sobre el territorio que no carece de complejidad. Este se concibe como un fenómeno multidimensional y no solo como un espacio físico, por lo que resulta indispensable conocer el entorno cultural y social en el que desembarcan las políticas “(...) más allá del barrio, la ciudad, la región. Es un espacio físico, pero también es mucho más: es el conjunto de vectores que incide sobre las personas y las familias" (MDSN, 2013). Asimismo, los territorios a los que se apunta se presentan - tanto en la normativa como en la representación de los agentes estatales - como espacios subordinados que disputan su representación "desde abajo".

Es decir, como zonas habitadas por sectores populares cuya pobreza se produce a consecuencia de una postergación del Estado, o bien, como resultado de la imposibilidad de acceder a este. Dichos espacios, como lo mencionó Juan17: "no figuran ni en los mapas,

17 Los nombres de las personas entrevistadas han sido modificados a fin de preservar su anonimato. 
ni en las estadísticas, son lugares olvidados por el Estado" (Juan, comunicación personal, coordinador nacional en el MDSN, /05/05/2016).

Pese a que en sus razonamientos y evaluaciones, los actores estatales reconocen que estas áreas se extienden ampliamente a lo largo del territorio argentino, simultáneamente sostienen que la "penetración estatal"18 sobre estos espacios requiere un conocimiento específico de la vida social $y$ del conjunto de interacciones que los constituyen como una realidad particular. Así, lo señala un entrevistado miembro del equipo del MDSN, quien trabajó en el "Plan Ahí" a lo largo de los años que tuvo vigencia:

(...) me parece que vos tenés que tener un conocimiento de la realidad y la única forma que tenés, que lo hemos hecho $y$ que se puede hacer, es con la gente. De otra forma no podés, por más que puedas mirar Google Maps. Yo siempre me acuerdo de una señora que una vez me dijo: 'mire, nosotros no existimos ni en el Google Maps, y era verdad, fui a googlear la localidad y no estaba'. No podés construir políticas públicas desde la ingenuidad, desde un manual de las buenas intenciones o desde un lugar de marketing (Operador del MDSN, comunicación personal, 05/05/2016).

Sin embargo, esas realidades específicas cuyo conocimiento es puesto en valor, y que solo la permanencia "ahí, en el lugar" hacía posible, podía referirse tanto a una Villa de la Ciudad Autónoma de Buenos Aires, a un barrio

$18 \quad$ La cuestión de la penetración estatal se presenta como un elemento recurrente desde los albores de la conformación del Estado Argentino. Así, por ejemplo, Oscar Oszlak da cuenta de que con la instauración del gobierno de Mitre se hace necesaria una descentralización del control, es decir, "la construcción de una "presencia" institucional permanente que fuera anticipando $y$ disolviendo rebeliones interiores y afirmando la suprema autoridad del Estado nacional” (1982, p.9). Dicha presencia no podía ser solo coercitiva sino también articuladora, consensual, material e ideológica. Lo dicho muestra que, desde la conformación del estado moderno argentino, la penetración estatal se constituye como un problema multifacético. del Conurbano Bonaerense, a un paraje o a un poblado rural del interior del país al cual solo es posible llegar "cruzando la frontera con Bolivia y volviendo a entrar"19; $y$ en algunos casos, incluso, se circunscribe al lugar sobre el que se asienta una comunidad originaria específica. En este sentido, como se verá a continuación, los espacios en los que desde la perspectiva de los actores "falta Estado" y a los que, por ende, debe aproximarse, revisten una gran heterogeneidad. En este sentido, ¿cómo se piensa la intervención del Estado Nacional sobre territorios diversos entre sí?

\section{LA HETEROGENEIDAD DE LOS TERRITORIOS Y LAS ORIENTACIONES DE LAS INTERVENCIONES ESTATALES}

Al respecto, en esta investigación es evidente una diferencia ostensible respecto al modo en que se interpretan y se problematizan los territorios. Por un lado, se encontrarían las áreas rurales del interior del país mientras que, por otro lado, estarían aquellos territorios inscriptos en los conglomerados urbanos de la AMBA. En el primer caso, el Estado tendría un grado de "poder infraestructural" bajo o nulo (Mann, 1997). Es decir, una incapacidad institucional del Estado Nacional para penetrar en sus territorios, efectuar intervenciones logísticas e implementar decisiones ${ }^{20}$.

Hay un Estado que "históricamente falta", que ha abandonado estos territorios debido a una improductividad e inviabilidad económica que les fue supuesta. Por ende, allí la llegada de la Nación se plantea como un intento de "penetrar" dichos territorios, de acortar distancias geográficas a fin de vincular a la comunidad con el aparato institucional. Esto se

$19 \quad$ Así, lo refirió uno de los entrevistados que trabajó como Coordinador Provincial del MDSN, al relatar los viajes que hacía en el interior del país.

20 En Las fuentes de poder social, Michael Mann (1997) diferencia dos dimensiones del poder estatal: el "poder despótico", que refiere al poder que poseen las élites estatales sobre la Sociedad Civil y el "poder infraestructural" que refiere a la capacidad de implementación del Estado central. Se trataría no de dos formas de poder sino, antes bien, de una forma que se desdobla en dos. 
materializa en la implementación de acciones tales como: la creación de caminos, viviendas rurales, la fomentación de economías solidarias y la asignación de recursos que permitan generar nuevas tramas económicas y sociales.

(...) Por ahí en Acambuco llega a la reunión y sale el enfermero. Le dice iay!', la recibe, alegría y demás, y llega con la gente de la provincia, municipio, qué sé yo. Dice 'ay, llegó la Nación', entonces ella ${ }^{21}$ le contesta 'está bien que soy gordita pero no soy tan grande como la Nación'. Entonces un poco esta idea de 'llegó la Nación' a lugares donde hacía 50 años que no había construcción de obra pública. La última vez fue la escuela que hizo Perón, en muchas localidades. Y lo digo con orgullo y también de pensar cuántos años de olvido en todos estos lugares donde vivían hermanos nuestros, fueron dejados a la mano de Dios, nunca mejor dicho. Entonces, vos representabas a la Mesa Nacional y estabas identificado con la cuestión nacional, pero eso no era un impedimento. No es que yo me venía con una bolsa de cartas o de cosas, sino que vos vas a trabajar junto con ellos (seudónimo, Coordinador Provincial del MDSN, comunicación personal, 05/07/2016).

A su vez, este aspecto se plantea como un desplazamiento sobre el carácter que adoptaron las políticas asistenciales implementadas durante la década de los 90, en las que habría primado un enfoque mercantilizador del bienestar social. Como lo planteó la entonces Ministra de Desarrollo Social, Alicia Kirchner: "Han estado a un paso de proponer una calificadora de riesgo que diga cuáles de los argentinos son sustentables y cuáles no. 0 tal vez lo hicieron, a juzgar por las políticas que algunos aplicaron cuando les tocó ser funcionarios" (MDSN, 2013a).

Sin embargo, como toda intervención de gobierno, el PA no se implementó de forma:

Se alude a la Directora Nacional del Plan Ahí.
(...) porque cuando algún pobre vive en las grandes ciudades de alguna manera siempre puede llegar a acceder a algún servicio, pero tenemos a argentinos $y$ argentinas en poblaciones remotas, que no figuran en ningún mapa, ni en ninguna encuesta ni estadística ${ }^{22}$ como nos contaba recién de la isla Apipé, o en pequeños pueblos del NOA $y$ del NEA, que no tienen ni siquiera las posibilidades de acceso a ningún tipo de bien y a ningún tipo de servicio porque no hay nada en su propio hábitat, $y$ pueden recorrer kilómetros y kilómetros sin encontrar nada, un hospital o una escuela o un dispensario (Cristina Fernández de Kirchner, Discurso de presentación del Plan Ahí, abril del 2008).

De esta manera, durante lo que se entiende como su etapa inicial, se produjeron los primeros esfuerzos de articulación política, especialmente entre la Ministra de Desarrollo Social y los gobernadores e intendentes de las distintas provincias del interior del país. En dicho momento, las intervenciones en el marco del plan abarcaron 223 pequeñas localidades rurales y parajes, y para el año 2009 alcanzaron a 434. Para fines de 2009, se amplía el número de localidades en las cuales se interviene, $y$ ya en septiembre de ese año, el PA cubre: 14 Provincias de las regiones Noroeste, Noreste, Centro y Cuyo; 144 barrios de 14 partidos del Conurbano Bonaerense; 5 de Resistencia y 2 de Concordia, con mayor índice crítico, tales como, asentamientos $y$ villas de emergencia (MDSN, 2009, p.24).

22 Como se mencionará más adelante, pese a lo manifestado por la entonces presidenta de la Nación $y$ por muchos funcionarios estatales, que sostienen que estas poblaciones no aparecen en ningún registro formal, señalando así la ausencia de Estado, las intervenciones en el marco del Plan Ahí se realizaron tomando en cuenta datos provenientes de la EPH, datos estadísticos regionales $y$, posteriormente, encuestas y evaluaciones específicas directamente vinculadas con la aplicación del Plan. Muchas veces se encontró que ambas ideas aparentemente contradictorias conviven en las representaciones de los informantes clave. 
No obstante, la instancia sucesiva (que tiene lugar desde el 2011 y que se considera como el período de consolidación del plan) se caracteriza por el proceso de ampliación y diferenciación de las áreas de incumbencia del Plan en que las intervenciones sobre distintos barrios del Conurbano Bonaerense $y$ Villas Miseria de la Capital Federal cobran una mayor centralidad ${ }^{23}$. Según el informe presentado en el MDSN, en junio de 2012, se inició el trabajo del PA en los siguientes barrios: La Cárcova, Independencia y Villa Cildañez y Villa 31 de la CABA.

Para septiembre de ese mismo año, se extendió nuevamente a otros barrios de municipios de la Provincia de Buenos Aires, tales como: Lanús, Avellaneda y Quilmes, y la Villa 21-24 y Zabaleta, de la Ciudad Autónoma de Buenos Aires (MDSN, 2014). En efecto, en el último informe oficial que se registra, producido por el Comité Nacional del programa Management of Social Transformation (MOST), se relevó que el PA estaba siendo implementado en 1400 pequeñas localidades y barrios, con el impulso de 812 Centros Integradores comunitarios (cIC) y la conformación de 1500 mesas de gestión a lo largo del país (UnESCo, 2015).

Ahora bien, las intervenciones que se dirigen hacia el interior del país y aquellas que recayeron sobre los espacios urbanos del AMBA, no se distinguen meramente en términos cuantitativos. La principal diferencia yace en que (en la percepción de las personas funcionarias y operarias del Plan) el problema central a resolver, en la Capital Federal y en el Conurbano Bonaerense, sería la dificultad que tienen las poblaciones de "acceder al Estado" en razón

23 En diciembre de 2010, se produjo la toma del Parque Indoamericano en el que un grupo de familias con necesidades habitacionales ocuparon un predio abandonado en el Sur de la Ciudad de Buenos Aires. Su desalojo violento produjo la muerte de 3 personas y una serie de desacuerdos entre el Gobierno de la Ciudad y el Gobierno Nacional en torno a la definición del conflicto y los modos de resolución. A partir de este momento, emergen distintos programas que focalizan la intervención sobre las villas de la Ciudad de Buenos Aires, tal es el caso de los programa de seguridad "Cinturón Sur" y "Centinela". de las complejidades de sus procesos y tramas burocráticas, así como de la guetización de los territorios urbanos empobrecidos. Es decir, en este caso la brecha entre las instituciones estatales $y$ los territorios afectados por distintas problemáticas sociales se interpreta como una distancia que no es tanto espacial como simbólica. Precisamente, es esta la distancia que el PA apunta a resolver; como se aprecia en un pasaje de la entrevista realizada a Lucía, quien trabajó para el Ministerio de Educación en la Villa 21-24 de Barracas:

Hay gente que pasa toda su vida sin DNI en los barrios. Una mamá, que tiene siete hijos y de los siete hijos solo dos tienen DNI. O porque sacó dos DNI y los otros nunca los gestionó, o porque los perdió, o porque se los llevó el agua en las inundaciones. No te olvides que en los barrios el agua viene de arriba y de abajo, entonces se te inunda la casa. Se te inunda la casa y se te mojan los papeles que tenías en un cajón, $y$ te quedaste sin partida de nacimiento, te quedaste sin DNI, lo que fuera. Esa mamá no sabe cómo sacar porque no tiene, no le llega la información. Si yo voy y le digo 'mirá mamá, esto es re fácil, se hace así y así', ahí se entera; pero yo tuve que ir hasta allá para que se entere, todo es mucho más complicado para ellos. Porque no tienen información, porque todo les parece más difícil, todo les cuesta más, porque si tienen que ir al centro a sacar el DNI... yo te explico, yo iba en taxi, me lo pagaba el Ministerio, esta gente tiene que salir para poder ir al centro (Operadora del Ministerio de Educación, comunicación personal, 03/10/2015).

La entrevista anteriormente citada permite inferir que la aproximación que el Estado busca producir mediante su instalación temporal en los territorios y la circulación de sus representantes ministeriales, supone un diagnóstico de "ciudad dual" (Young, 2008). En esta idea de ciudad excluyente, las barreras serían casi imposibles de cruzar con regularidad, ocasionando que los pobres se encuentren moral y 
físicamente apartados. No obstante, sin negar el establecimiento de barreras de exclusión, se entiende que tales parámetros físicos tienden a exagerar la idea del aislamiento social y a eludir que las "comunidades" trascienden cotidianamente las demarcaciones físicas y comparten extensamente los valores generales de la sociedad.

La distinción que se produce en los diagnósticos y caracterizaciones de los territorios antes mencionados permite comprender, además, que el desembarco del Estado en el marco del PA se orientó a atender problemas específicos en virtud de la particularidad de los espacios sociales. Si para el caso de las zonas rurales la importancia del PA radicaba en la extensión de la capacidad infraestructural del Estado, para el caso de las Villas de la Ciudad de Buenos Aires, la intervención estatal se propondría franquear el aislamiento y la desvinculación con la trama institucional del Estado (vinculada a la pobreza y marginalidad producida a efectos del modelo económico implementado en los años 90). Es decir, la importancia de aproximar al Estado radica en la morigeración del deterioro de las condiciones de vida, expresadas en la incapacidad de acceso material y simbólico a las instituciones y agencias estatales.

En suma, se puede afirmar que en el Plan Nacional que se ha analizado predominó una delimitación territorial de la pobreza cuya causa es leída, principalmente, como un efecto de la "ausencia del Estado" sobre determinadas zonas. Asimismo, se entiende que en el PA los territorios de la pobreza son heterogéneos porque las exclusiones de las poblaciones que los habitan se distinguen; o bien, por haber quedado fuera de "el desarrollo", de la "relación salarial normal" o de formas deseables de “integración social" (Grondona, 2014, p.192). La enorme diversidad que albergan estos territorios entre sí puede ser equiparada solo en razón de que su condición de pobreza es conceptualizada como "vulnerabilidad" o "riesgo". Sin embargo, en este caso de estudio particular, esta vulnerabilidad es concebida como resultado de una falta estatal históricamente producida y que, como se vio, no es la misma en todos los casos.

Por otra parte, como se verá a continuación, el análisis permitió distinguir algunas tensiones en la dinámica de implementación que apuntaba a la coordinación de distintos niveles de gobierno. Estos conflictos se vinculan no solo a la heterogeneidad de los grupos y poblaciones o de los problemas prioritarios a ser atendidos en cada uno de los territorios, sino a la capitalización política del plan como dispositivo de intervención territorial que construye presencia estatal.

\section{TENSIONES Y DISPUTAS EN TORNO A LA CONSTRUCCIÓN DE LA PRESENCIA ESTATAL}

La selección de las áreas de incumbencia $y$ el acceso de las distintas agencias estatales a estas, fueron puntos sobre los que se presentaron desacuerdos $y$ tensiones a nivel político $\mathrm{e}$ interinstitucional. Estas discrepancias se produjeron, tanto entre los agentes del MDSN y los agentes estatales de los restantes ministerios, como entre los funcionarios del Estado Nacional y las autoridades políticas locales, en torno a la pertinencia de la instalación de la "Nación" (como usualmente se le denominó) y a la definición de los espacios prioritarios a intervenir.

Las tensiones que se registraron a lo largo de las entrevistas realizadas permiten afirmar que lo entendido por los actores como el "desembarco del Estado Nacional", vale decir, el despliegue de sus diversas acciones sobre el terreno, produjeron disputas por la capitalización de la presencia estatal. Siguiendo a Sack (1986), se entiende que cada instancia territorial involucra un modo de implementar el control tanto sobre el acceso al área como sobre las cosas dentro de ella. Sin embargo, resulta relevante destacar que lo que se presenta como objeto de conflicto en esta intervención gubernamental no fue solo la distribución de bienes y recursos que habilita mediante la política asistencial — tradicionales objetos de enfrentamiento político (Perelmiter, 2016) - , sino "la puesta en escena del acto asistencial" y, por tanto, la presencia del Estado en un territorio dado. 
Aunque para los agentes del MDSN el uso de instrumentos estadísticos, tales como censos $y$ encuestas, funcionaron como herramientas aseguradoras de objetividad (que permitiría sortear intereses político-partidarios entre intendentes, gobernadores y el Estado Nacional) estos intereses, en muchos casos, habilitaron $u$ obturaron efectivamente la implementación y/o permanencia del plan en un espacio asignado. La disputa de los territorios por el gobierno, se expresó, entonces, en los intentos de capitalizar la presencia estatal $y$ de hacerse, a partir de ello, de un "capital territorial" que permitiera reafirmar liderazgos políticos.

Por ejemplo, en el caso del Barrio Independencia, el intendente pidió ir ahí porque no tenía trabajo territorial "yo acá quiero estar porque necesito construir presencia territorial para atender las demandas que tengo acá, en Corina tengo presencia territorial, política e institucional, pero necesito fortalecerla" (seudónimo, Coordinador General del Ministerio de Educación de la Nación, comunicación personal, 05/08/2016).

No pudimos generar institucionalidad, ni fortalecer la institucionalidad, ni saldar heridas previas que había, inter-institucionales. Porque el Plan Ahí tenía una debilidad de base que era que no estaba claro quién era, cuál era el liderazgo institucional y político en el territorio. Vos ibas a un municipio donde en algunos casos estaba referenciada la autoridad municipal, en otros casos no, y eso ya te generaba una situación distinta, porque en el caso de que la autoridad municipal estuviera presente los ministerios se retraían un poco y trataban de coordinar con la autoridad municipal. Cuando la autoridad municipal no estaba presente, los ministerios tenían un rol mucho más activo en toda la canalización de las demandas (seudónimo, Operador del Ministerio de Educación, comunicación personal, 13/12/2015).
Esto cobra sentido en tanto que el "Plan Ahí" no solo atendió necesidades sociales urgentes en un tiempo muy breve $y$ sin mayores mediaciones burocráticas, sino que lo hizo mediante un despliegue visual ostensible.

(...) vos veías en una plaza de un pueblito por 7 días, 15 días, 20 días, camiones del Ministerio de Salud, del Ministerio de Educación, de ANSES, que se instalaban todos juntos. En un día te podías hacer el DNI, los anteojos, que te revisen los médicos, hacer un taller de género, eso a la gente le gustaba mucho, era como oro en los pueblos donde casi no hay médicos, hacerse los anteojos, que te revisen, tenía mucha demanda (Operadora del Ministerio de Salud de la Nación comunicación personal, 12/09/2016).

Por ello, la posibilidad de implementar efectivamente el Plan requería de la validación $y$ el consenso previo de las autoridades locales y de la existencia de referentes comunitarios que habilitaran el acceso al territorio, especialmente en barrios $y$ villas del AMBA en los que el grado de organización política era mayor. Por otro lado, desde la perspectiva de los agentes de los restantes ministerios, se destacan las menciones acerca de los intereses $y$ las rispideces políticas que produjo la implementación del Plan.

En concordancia, se encontró en casi todas las entrevistas que si bien, las tensiones políticas entre el Gobierno Nacional y algunas autoridades locales $y$ provinciales estuvieron presentes desde los inicios de su implementación, estas se agudizaron en la última etapa de desarrollo del programa. En este último momento, que se desarrolló en el año 2014, se produjo un retraimiento $y$ algunas modificaciones en lo referido a su implementación, disminuyendo tanto la frecuencia de las intervenciones, es decir, la presencia de agencias con participación activa y de recursos asignados, como las áreas de intervención, especialmente en el orden del AmBA.

Desde la perspectiva de los actores estatales esto se debió, por un lado, a diferencias con las autoridades políticas cuyas filiaciones partidarias (opuestas al Gobierno nacional de 
turno) impactaron en la capacidad de articulación y coordinación; a la inexistencia de equipos institucionalizados fuera del Ministerio de Desarrollo Social ${ }^{24}$; $y$ por otro lado, a una crisis económica $^{25}$ que imposibilitaba la ejecución de un plan que, por sus características, requirió una gran asignación presupuestaria, tanto para el transporte de bienes $y$ servicios como para la permanencia de los equipos técnicos ${ }^{26}$. Aunque en el interior del país, este plan continuó desarrollándose con una regularidad mayor a la de Capital Federal y del Conurbano Bonaerense, con la llegada de Mauricio Macri a la Presidencia de la República, a fines del año 2015, y pese a no existir un comunicado oficial que lo anunciase, el Plan quedó suspendido y los equipos fueron desintegrados y reasignados a otros programas, cuando no despedidos en el marco del proceso de "revisión" 27 o achicamiento del tamaño de la administración pública nacional ${ }^{28}$.

El resquebrajamiento de la legitimidad con la que contaba la coordinación y el

24 Como se mencionó anteriormente, si bien existían equipos designados dentro de cada ministerio, muchos de los agentes ministeriales debían sumar a sus tareas habituales el trabajo territorial, es decir, gran parte de ellos no se constituyeron como equipos con una dedicación exclusiva al "Plan Ahí".

Las personas entrevistadas hacen referencia a la desaceleración de la economía argentina, que pasa de un crecimiento de un 2,9\% en el año 2013 a una expansión de $0,5 \%$ en el 2014, con un desmejoramiento de los principales indicadores económicos (CEPAL, 2015). Información disponible en: http://repositorio.cepal.org/bitstream/handle/11362/38713/Argentina_es.pdf;jsessionid=22B2C 8FD8BDB479ED33DF494B022C040? sequence $=20$

26 A esto se suma que, como se mencionó, el "Plan Ahí" no contaban con un presupuesto propio, sino que dependía de la asignación de recursos de cada agencia ministerial.

27 Véase: http://www.lanacion.com.ar/1935275-el-gobierno-hara-revision-integral-planta-empleados

28 Actualmente, se encuentra en vigencia un programa de características similares que funcionaría como una reconfiguración del "Plan Ahí": "El Estado en tu barrio" (Decreto 665/2016). Su estudio y el análisis de sus continuidades o trasformaciones queda pendiente para futuras investigaciones. descenso de la vitalidad que la caracterizó durante sus primeros años, se puso de manifiesto en los cuestionamientos sobre la definición de las áreas de incumbencia. La selección de espacios fue percibida (por gobernadores e intendentes, incluso por algunos de los funcionarios ministeriales) como una herramienta "estratégica" en la cual primó cierta voluntad de capitalización electoral frente a los comicios próximos en octubre de 2015 . Esto habría evidenciado en la relegación de otros espacios que requerían una atención estatal más urgente y que, finalmente, permanecieron excluidos del radio de acción del Plan pero, a su vez, en que los actos de cierre de los operativos oficiaran a modo de campaña electoral.

\section{REFLEXIONES FINALES}

En Argentina, la impugnación al modelo neoliberal se manifestó en diversos órdenes y en distintos niveles de la política pública luego del año 2003. Específicamente, el objetivo de esta indagación empírica fue tomar una pequeña arista de esta cuestión para reflexionar acerca de los modos en los que se concibieron, desde la narrativa estatal, las intervenciones territoriales en el campo de la política asistencial y sobre los objetivos estratégicos a los que esta apuntó.

No obstante, antes que definiciones preestablecidas, se indagó la naturaleza del Estado en el territorio, el sentido que los actores estatales le asignaron a esas intervenciones $y$ las tensiones que habitaron las permanencias episódicas del Estado Nacional en los distintos espacios sociales sobre los que desembarcó el "Plan Ahí". En este sentido, se encontró una diferencia notable entre la forma en la que se problematizan e intervienen los territorios del interior del país, (especialmente los poblados y las áreas rurales), respecto de los territorios y las acciones sobre los conglomerados urbanos del AMBA.

Al respecto, se señala que en los primeros lugares, las intervenciones apuntan a revertir el bajo o nulo grado de "poder infraestructural" que presenta el Estado. Esto es, una ausencia "histórica" de larga procedencia que habría producido una asimetría geográfica en el plano económico y social. Por ello, los intentos 
por parte del Estado Nacional por "penetrar" las zonas más distantes de Buenos Aires buscaron acortar distancias espaciales $y$ vincular estas áreas con el aparato institucional del Estado central a fin de reactivar sus tramas productivas. Por otra parte, para el caso de las zonas del АMBA, las intervenciones del "Plan Ahí" busca trasponer la fragmentación urbana $y$ la marginalidad producida por el retiro del Estado durante los años 90. Es decir, si en ambos casos se reconocen territorios heterogéneos, predomina una delimitación de la pobreza cuya causa es leída, en la racionalidad de los actores estatales, como producto de ausencias del Estado que tienen procedencias históricas y políticas específicas, a partir de lo cual su retorno deviene urgente.

Se señala, a su vez, cómo con el desembarco del Estado Nacional y el despliegue de las diversas acciones que implementaron durante los años en los que tuvo vigencia el dispositivo, las disputas por la capitalización de la "presencia estatal" exceden a la distribución de los bienes y recursos que gestionan los diversos programas que comprendió el "Plan Ahí". En este sentido, los enfrentamientos políticos giraron en torno a la puesta en escena del "acto asistencial", así como, a la obtención de un "capital territorial", es decir, a la vinculación con los actores territoriales y al saber sobre sus prácticas y dinámicas que permitían la inserción del Estado en un espacio dado.

Ahora bien, a lo largo de este trabajo, se ha dado cuenta de qué manera se reflexionó en torno a los territorios en las intervenciones que tuvieron lugar en el marco del Plan. Estos razonamientos hicieron hincapié en la dimensión "negativa" del territorio (Varela, 2010) que enfatiza la caracterización de la vulnerabilidad, los problemas de desarrollo y la desvinculación de los distintos espacios sociales con las tramas institucionales del Estado. No obstante, aunque este tema excedió el objeto de este artículo, es necesario señalar que se encontró que la definición de lo territorial está simultáneamente vinculada a una noción de la territorialidad que, se entiende, es más bien productiva. Se trata de la idea de "comunidad" que se plantea en el Plan.
El acoplamiento territorio-comunidad es tal que, en algunos casos, puede pensarse en una homologación entre ambas nociones. La remisión a la comunidad y, específicamente, la interpelación a la "comunidad organizada" apelaron al elemento activo y organizacional de la población o, más precisamente, de las organizaciones político-sociales locales, que no subordinó ni el componente político ni el entramado histórico a partir del cual tiene lugar la participación de los sectores populares ${ }^{29}$.

Por otra parte, el programa que se estudió se enuncia como una "metodología", como un modelo organizador de la práctica estatal que, a partir de la permanencia de los agentes estatales en los territorios empobrecidos, objetiva la idea de un "retorno del Estado Nacional", "cercano" y "presente". Con esto, no se afirma que las estrategias de gobierno se establezcan de modo unitario para todo el campo estatal, que alberga numerosas agencias y en la que intervienen una diversidad de actores. No obstante, dichas intervenciones dan cuenta del proyecto por parte del Estado Nacional de recuperar un lugar central en la definición del sentido de la política social y asistencial - por oposición al peso que tuvieron los gobiernos locales durante los años 90 - pero, a su vez, de producir algunos desplazamientos en torno a las racionalidades políticas que guiaron las intervenciones por esos años.

Pese a que la enunciación de un "retorno del Estado Nacional" bien podría reificar la idea de una ausencia previa de Estado, más que impugnar una modalidad específica de gobierno que tuvo como efecto la profundización de la desigualdad social. Los intentos por redireccionar las intervenciones de abordaje territorial expresan, a su vez, reflexiones en torno a las tramas históricas que producen los distintos espacios sociales

29 Esta cuestión ha sido abordada con mayor detenimiento en el artículo: "Transformaciones en el gobierno de la "comunidad" en Argentina: del empowerment a la comunidad organizada". Delito y sociedad. Revista de Ciencias Sociales. Numero 45 (en prensa). 
a los que apuntaron las intervenciones, así como, un énfasis en la responsabilización del rol estatal en la producción o morigeración de la desigualdad social visible en los territorios.

\section{REFERENCIAS}

Ayos, E. (2017). Asistencia, trabajo y peligrosidad. La política social y la cuestión de la seguridad en la Argentina de la última década. Gaceta Laboral, 23 (1), 08-31.

Catenazzi, A. (2015). "Para conceptualizar el territorio en salud". En M. Chiara (Comp.), Gestión territorial integrada para el sector salud (pp.37-47). Los Polvorines: Ediciones UNGs.

Catenazzi, A. y Quintar, A. (2009). Presentación. En Catenazzi, A., Quintar, M., Cravino, C., Da Representacao, N. y Novick, A. (eds.). El retorno de lo político a la cuestión urbana. Territorialidad y acción pública en el Área Metropolitana de Buenos Aires. Buenos Aires: Ediciones UNGS.

CEPAL (2015). Estudio económico de América Latina y el Caribe. Recuperado de https://repositorio.cepal.org/bitstream/handle/11362/38713/Argentina_ es.pdf;jsessionid=22B

Clemente, A. (2013). Pobreza y Planificación Social. El territorio como variable de integración y movilidad social. En R. Castronovo (Coord.), Políticas Sociales en debate. Los nuevos temas de siempre. Buenos Aires: Eudeba.

Danani, C., y Hintze, S. (2014). Protecciones y desprotecciones II. Problemas y debates de la seguridad social en la Argentina. Buenos Aires: Ediciones UNGS.

Dallorso, N. (2016). Un nuevo escenario para las prácticas de control social: los programas de transferencias monetarias condicionadas en Latinoamérica. Delito y Sociedad, 2(30), 103-113.

Fernández de Kirchner, C. (11/04/2008). Lanzamiento del Plan de Abordaje Integral. Casa Rosada. Recuperado de https://www.casarosada.gob.ar/informacion/archivo/16903-blank-52631605
Foucault, M. (2006). Seguridad, Territorio y Población. Buenos Aires: Fondo de Cultura Económica.

Giavedoni, J.G. (2012). Del Estado en crisis a la crítica del Estado: Diálogo en torno a la perspectiva del Estado y la gubernamentalidad en el análisis de la nueva cuestión social en América Latina. Revista mexicana de ciencias politicas y sociales, 57 (214), 89-109.

Giavedoni, G. (2012). Gobernando la pobreza: la energía electrica como dispositivo de gestión de los sectores populares. Rosario: Homo Sapiens Editores.

Grassi, E. (2003). Politicas y problemas sociales en la sociedad neoliberal. La otra década infame (I). Buenos Aires: Espacio Editorial.

Grondona, A. L. (2014). Saber de la pobreza. Discursos y subclases en la Argentina entre 1956-2006. Buenos Aires: Centro Cultural de la Cooperación Ediciones.

Hintze, S. y Costa, M. (2011). La reforma de las asignaciones familiares 2009: aproximación al proceso político de la transformación de la protección. Protecciones y desprotecciones: la seguridad social en la Argentina 1990-2010. Los Polvorines: Universidad Nacional de General Sarmiento.

Jefatura de Gabinete de la Nación. (2004). Memoria detallada del Estado de la Nación, año 2003.

Jefatura de Gabinete de la Nación. (2010). Los desafíos de la coordinación y la integralidad de las políticas y gestión pública en América Latina.

Jefatura de Gabinete de la Nación. (2010). Memoria detallada del Estado de la Nación, año 2009.

Maneiro, M. (2015). Representaciones sociales sobre el Programa Argentina Trabaja en las clases populares urbanas. Revista Katálysis, 18 (1), 62-73.

Mann, M. (1997). Las fuentes del poder social, II. El desarrollo de las clases y los Estados Nacionales 1760-1914. Madrid: Alianza.

Manzanal, M. (2016). El desarrollo desde el poder $y$ el territorio. En A. Rofman 
(Comp.), Participación, políticas públicas y territorio. Apuntes para la construcción de una perspectiva integral. Buenos Aires: Ediciones UNGS.

Merklen, D. (2000). Vivir en los márgenes: la lógica del cazador. Notas sobre la sociabilidad y cultura en los asentamientos del Gran Buenos Aires hacia fines de los 90. En M. Svampa (editora). Desde abajo: La transformación de las identidades sociales (pp 81-119). Buenos Aires: Biblos.

Ministerio de Desarrollo Social de la Nación (MdsN). (2007). La Bisagra. Politicas sociales en acción. Recuperado de http:// www.fts.uner.edu.ar/catedras03/politica_social/documentos/La_Bisagra_ Politicas_Sociales_en_accion.pdf

Ministerio de Desarrollo Social de la Nación (MDSN). (2009). Rendimos Cuentas. Diciembre 2007-mayo 2009. Recuperado de http://www.desarrollosocial.gob.ar/ biblioteca/rendimos-cuentas-diciembre2007-mayo-2009/

Ministerio de Desarrollo Social de la Nación (MDSN). (2010a). Políticas Sociales del Bicentenario I. Recuperado de http:// www.desarrollosocial.gob.ar/biblioteca/ politicas-sociales-del-bicentenario-i-2/

Ministerio de Desarrollo Social de la Nación (MDSN). (2010b). Politicas Sociales del Bicentenario II. Recuperado de http:// www.desarrollosocial.gob.ar/biblioteca/ politicas-sociales-del-bicenten

Ministerio de Desarrollo Social de la Nación (MDSN). (2013a). Los Derechos Sociales son Derechos Humanos. Recuperado de http://www.desarrollosocial.gob.ar/ biblioteca/los-derechos-sociales-sonderechos-humanos/

Ministerio de Desarrollo Social de la Nación (MDSN). (2013b). Recuperación de experiencias con abordaje comunitario. Recuperado de http://www.desarrollosocial.gob.ar/biblioteca/recuperacion-deexperiencias-de-abordaje-comunitario/

Ministerio de Desarrollo Social de la Nación (MDSN). (2013c). Territorios. Huellas y horizontes de una nueva institucionalidad de políticas sociales. Recuperado de https://www.desarrollosocial.gob.ar/ wp-content/uploads/2015/05/6-Territorios1.pdf

Ministerio de Desarrollo Social de la Nación (MDSN). (2015). Políticas públicas con impacto social. Recuperado de http:// www.desarrollosocial.gob.ar/biblioteca/ politicas-publicas-con-impacto-social/

Ministerio de Desarrollo Social de la Nación (mDSN). (s/f). Plan Ahí tríptico. Recuperado de http://www.desarrollosocial.gob.ar/enlace/plan-ahi-folleto/

Ministerio de Desarrollo Social de la Nación (MDSN) (s/f). Participar es tu derecho folleto. Recuperado de http://www.desarrollosocial.gob.ar/enlace/participar-estu-derecho-folleto/

Ministerio de Educación de la Nación. (2014). Informe Plan Nacional de Abordaje Integral. Recuperado de http://www.sipi. siteal.iipe.unesco.org/sites/default/files/ sipi_intervencion/plan_ahi.pdf

Ministerio del Interior de la Nación. (2004). Programa Mi Pueblo. Por la recuperación de pueblos y comunidades en todo el territorio nacional.

Murillo, S. (2008). Colonizar el dolor: La interpelación ideológica del Banco Mundial en América Latina. El caso argentino desde Blumberg a Cromañon. Buenos Aires: Consejo Latinoamericano de Ciencias Sociales-CLAcso.

Oszlak, O. (1982). Reflexiones sobre la formación del Estado y la construcción de la sociedad Argentina. Desarrollo Económico Revista de Ciencias Sociales, (21) 84, Buenos Aires, Argentina.

Perelmiter, L. (2016). Burocracia plebeya. La trastienda de la asistencia social en el Estado Argentino. San Martín: UnSAM EDITA.

Piva, A. (2015). Economía y política en la Argentina Kirchnerista. Buenos Aires: Batalla de Ideas.

Rofman, A. (2016). Introducción. Rofman, A. (comp.). Participación, políticas públicas y territorio: aportes para la construcción de una perspectiva integral, (pp.9-27). Buenos Aires : Ediciones UNGS. 
Rofman, A. (2016). Introducción. En Participación, políticas públicas y territorio: aportes para la construcción de una perspectiva integral (pp. 9-27). Buenos Aires: Ediciones UNGS.

Rose, N. (2007). ¿La muerte de lo social? Re-configuración del territorio de gobierno. Revista Argentina de Sociologia, (8), 113-152.

Sack, R. (1986). Human territoriality: its theory and history. Cambrige: Cambrige University Press.

Servio, M. (2015). Cuestión social y constitución del sujeto receptor en la Asignación Universal por Hijo para Protección Social. Revista Katálysis, 18 (2), 231-238.

Soldano, D. y Andrenacci, L. (2006). Problemas de política social en la Argentina contemporánea. Los Polvorines: Prometeo Libros-UNGS.

Svampa, M. (2005). La sociedad excluyente. Buenos Aires: Siglo XxI.

Unesco y Comité Nacional Most. (2015). Radiografías de las políticas sociales del Siglo XXI. Las miradas populares. Argentina: Ministerio de Desarrollo Social.
Varela, P. (2010). Los límites del territorio. Una hipótesis sobre la tesis de la "territorialización de la política." En Massetti, A, Villanueva, E y Gómez, M. (comp.). Movilizaciones, protestas e identidades politicas en la Argenina del bicentenario (pp. 447-471). Buenos Aires: Nueva Trilce.

Vasilachis de Gialdino, I. (2015). Estrategias de Investigación Cualitativa. Barcelona: Gedisa Editorial.

Vommaro, G. (2011). Los pobres y la pobreza como dominio experto: contribuciones a una socio-historia. Saber lo que se hace: expertos y politica en Argentina. Buenos Aires: Prometeo Libros.

Young, J. (2008). Merton con energía, Katz con estructura. La sociología del revanchismo y la criminología de la trangresión. Delito y sociedad. Revista de Ciencias Sociales, (25), 63-87.

Fecha de ingreso: 10/07/2018 Fecha de aprobación: 07/11/2018 